\title{
RESISTANT TO ATHEROSCLEROSIS
}

${ }^{1}$ Mustafa Zakkar, ${ }^{1}$ Hera Chaudhury, ${ }^{1}$ Gunhild Sandvik, ${ }^{1}$ Karine Enesa, ${ }^{1}$ Le Anh Luong, ${ }^{1}$ Simon Cuhlmann, ${ }^{1}$ Justin C Mason, ${ }^{2}$ Rob Krams, ${ }^{3}$ Andrew R. Clark, ${ }^{1}$ Dorian O Haskard and ${ }^{1}$ Paul C Evans

${ }^{1}$ BHF Cardiovascular Sciences Unit, National Heart and Lung Institute and ${ }^{2}$ Department of Bioengineering and the ${ }^{3}$ Kennedy Institute of Rheumatology Division, Imperial College London, UK

Address for correspondence:

Dr. Paul C Evans

Senior Lecturer

BHF Cardiovascular Sciences Unit

National Heart and Lung Institute

Imperial College London

Hammersmith Campus

Du Cane Road

London W12 ONN

UK

Tel.: +44 (0)20-83831619

Fax: $+44(0) 20-83831640$

paul.evans@imperial.ac.uk 


\section{ABSTRACT}

Introduction Regions of the arterial tree exposed to unidirectional laminar flow, which exerts high shear stress, are protected from endothelial cell (EC) activation, inflammation and atherosclerosis. Pro-inflammatory mediators (e.g. lipopolysaccharide (LPS)) activate p38 and JNK mitogen-activated protein (MAP) kinases by phosphorylation, which induce pro-inflammatory molecules including VCAM-1 in EC. We assessed whether regions of the arterial tree exposed to high shear were protected from pro-inflammatory activation by suppression of p38 and JNK. Methods Wild-type C57BL/6 and MAP kinase phosphatase- $1^{-/-}\left(\mathrm{MKP}^{-1^{--}}\right)$mice were studied. Expression levels of VCAM-1 and phosphorylated forms of p38 and JNK in EC were measured by en face staining in a region of the murine aorta that is exposed to relatively low shear and is pre-disposed to atherosclerosis (lesser curvature of arch) and in a region that is exposed to high shear and is protected (greater curvature). The expression of MKP-1 was assessed in human umbilical vein EC (HUVEC) exposed to high shear stress or to static conditions by comparative real-time PCR, immunofluorescence staining and Western blotting. Results We observed that phosphorylation of p38 and JNK and expression of VCAM-1 in EC occurs constitutively and can be enhanced by LPS treatment in the lesser curvature in wild-type mice. By contrast, p38 and JNK activities and VCAM-1 expression were suppressed in the greater curvature. We observed using cultured EC that high shear (12 dynes $/ \mathrm{cm}^{2}$ for $24 \mathrm{~h}$ ) induced MAP kinase phosphatase-1 (MKP-1), a molecule that inactivates p38/JNK by dephosphorylation. Similarly, MKP-1 was expressed at high levels in EC exposed to high shear in the greater curvature of the aorta but at relatively low levels in the lesser curvature. We examined the function of MKP-1 in aortic EC using MKP-1 ${ }^{-/-}$mice. En face staining revealed that EC in the greater curvature of MKP-1 ${ }^{-/}$ mice were sensitive to LPS treatment as p38 and JNK were phosphorylated and VCAM-1 was induced. Conclusion MKP-1 is expressed by EC in protected regions of the aorta where it suppresses EC activation. Our findings reveal a novel mechanism to explain protection from vascular inflammation and atherosclerosis in arteries exposed to high shear. 


\section{INTRODUCTION}

Atherosclerosis is a chronic inflammatory disease of arteries ${ }^{1}$. Early lesions (fatty streaks) contain monocytes and T-lymphocytes which are recruited from the circulation by adhesion to activated vascular endothelial cells (EC) ${ }^{2}$. Microbial products (e.g. LPS, endotoxin), which may be circulating due to intercurrent infections ${ }^{3}$, activate both NF- $\kappa B$ and mitogen activated protein (MAP) kinase signalling pathways which co-operate to induce pro-inflammatory proteins such as VCAM- $1^{4,5}$. JNK and p38 MAP kinases play an important role in vascular inflammation ${ }^{6-10}$. They are activated in EC in response to pro-inflammatory stimuli by dual phosphorylation of Thr-X-Tyr motifs within the phosphorylation loop ${ }^{10}$. Active forms of JNK and p38 phosphorylate transcription factors belonging to the AP-1 superfamily (including c-Jun, ATF2, c-fos) which subsequently form complexes with NF$\kappa \mathrm{B}$ at the promoters of pro-inflammatory genes including VCAM-1 ${ }^{11-13}$. Activation of $\mathrm{p} 38$ also regulates VCAM-1 at a post-transcriptional level by enhancing message stability ${ }^{14}$. Pro-inflammatory signaling also induces MKP-1, a negative regulator which inactivates p38 and JNK by removing phosphate groups from Tyr and Thr residues ${ }^{15}$.

Atherosclerosis occurs at distinct sites of the arterial tree located near branches and bends which are exposed to abnormal hemodynamics, whereas arteries with uniform geometries are relatively protected $^{16}$. For example, the lesser curvature of the murine aorta contains a region that has a high probability of developing atherosclerosis (called the HP site) whereas the greater curvature has a low probability of atherogenesis (LP site) ${ }^{17,18}$. Recent studies revealed that pro-inflammatory activation of EC is reduced in LP regions compared to HP regions, thus providing a potential explanation for the differing susceptibilities of these sites to atherosclerosis ${ }^{17,18}$.

Blood flow exerts shear stress (mechanical drag) on vascular endothelium, which varies in time, magnitude and direction according to vascular pulsatility and anatomy. Shear stress alters the physiology of endothelial cells (EC) which respond to it via mechanosensory receptors expressed at 
their surface that convert mechanical forces into numerous biochemical signals ${ }^{19,20}$. Several lines of evidence suggest that the spatial distribution of vascular inflammation and atherosclerosis in the arterial tree is regulated by shear stress. Firstly, the magnitude of shear stress at arterial walls is inversely correlated with their susceptibility to atherosclerosis. For example, analysis of the murine aorta by computational fluid dynamics revealed that regions exposed to high mean shear are protected from atherosclerosis whereas regions exposed to low shear are not ${ }^{16-18,21,22}$. Secondly, it has been shown that inflammation and atherosclerosis can be induced in murine carotid arteries by the application of a flow-altering device that generates spatial and temporal oscillation of the shear stress field $^{23}$. Finally, prolonged high shear exerts several protective effects on cultured EC including inhibition of the cell cycle $^{24}$, promotion of viability ${ }^{20}$ and suppression of pro-inflammatory activation $^{22,25-27}$.

The molecular mechanisms underlying the spatial distribution of vascular inflammation and atherosclerosis are uncertain. Here we demonstrate that MKP1 is expressed by EC in protected regions of the aorta exposed to high shear stress where it plays an essential role in the suppression of p38 and JNK activation and inhibition of VCAM-1 expression. 


\section{MATERIALS AND METHODS}

Animals Male C57BL/6 mice between 2 and 3 months of age were used. The MKP-1 knockout mouse strain (MKP-1 $1^{-/-}$(C57BL/6)) used in this study was obtained from Bristol-Myers Squibb (New York, USA). All experiments were performed within guidelines set out by the Federation of European Laboratory Animal Science Associations.

Reagents and antibodies Human recombinant TNF $\alpha$ and LPS (R\&D, Minneapolis, MN, USA) and anti-phosphop38 Tyr180/Thr182 (Cell signalling Technology, Massachusetts, USA), anti-phoshoJNK Tyr183/Thr185 (Cell signalling Technology, Massachusetts, USA), anti-p38 (Cell signalling Technology, Massachusetts, USA), anti-JNK (Cell signalling Technology, Massachusetts, USA), antiCD31-FITC (BD Biosciences Pharmingen, Oxford, UK) and anti-MKP-1 (Santa Cruz, USA) antibodies were obtained commercially. Other reagents were purchased from Sigma Aldrich (St. Louis, USA) unless otherwise stated.

En face staining The expression levels of specific proteins were assessed in EC at regions of the lesser curvature (HP site) and greater curvature (LP site) of murine aortae by en face staining as described previously ${ }^{17}$. The physiology of vessels in vivo was assessed using aortae that were perfusion-fixed with $2 \%$ formalin and then harvested. Alternatively, physiological responses of vessels were assessed ex vivo using aortae harvested from animals that were killed by $\mathrm{CO}_{2}$ inhalation. In the latter case, aortae were cut longitudinally along the greater curvature to expose the endothelial surface. They were then bathed in cell culture medium for varying times either in the presence or absence of pro-inflammatory stimuli and then fixed with $2 \%$ formalin. Fixed aortae from in vivo or ex vivo experiments were tested by immunostaining using specific primary antibodies and fluorescent secondary antibodies. Stained vessels were then mounted prior to visualization of endothelial surfaces en face using confocal laser-scanning microscopy (LSM 510 META; Zeiss, Oberkochen, Germany). EC were identified by co-staining using anti-CD31 antibodies conjugated to the fluorophore FITC 
(Invitrogen, Carlsbad, USA). Nuclei were identified using a DNA-binding probe with far-red emission (Draq5; Biostatus, Leicester, UK). Isotype-matched monoclonal antibodies raised against irrelevant antigens or pre-immune rabbit sera were used as experimental controls for specific staining (Supplementary Fig. 1). The expression of particular proteins at each site was assessed by quantification of fluorescence intensity for multiple cells (at least 100 per site) using LSM 510 software (Zeiss, Oberkochen, Germany).

Endothelial cells and exposure to flow Human umbilical vein endothelial cells (HUVEC) were collected using collagenase and cultured as described previously ${ }^{26}$. Confluent HUVEC cultures were exposed to high shear (12 dynes/ $\mathrm{cm}^{2}$ ) unidirectional laminar flow for $24 \mathrm{~h}$ using a parallel-plate flow chamber (Cytodyne, La Jolla, CA, USA) as described previously ${ }^{26}$.

Comparative real time PCR Transcript levels were quantified by comparative real-time PCR using gene-specific primers for MKP-1 (sense, 5'-CAGCTGCTGCAGTTTGAGTC-3'; antisense, 5'AGGTAGCTCAGCGCACTGTT-3’), MKP-2 (sense, 5’-TGCAAGGTAGCATGATGAGC -3’; antisense, $\quad$ 5’- $\quad$ TGAGCCTTGGCAACATAGTG-3’), $\quad$ MKP-3 (sense, 5’GGGCAAGAACTGTGGTGTCT-3’; antisense, 5'AGCAGCTGACCCATGAAGTT-3'), and $\beta$ actin (sense, 5'-CTGGAACGGTGAAGGTGACA-3'; AAGGGACTTCCTGTAACAATGCA-3'). Total RNA was extracted and reverse transcribed as described previously ${ }^{26}$. Real-time PCR was carried out using the iCycler system and SYBR green master mix (Biorad, Hercules, USA) according to the manufacturers' instructions. Reactions were incubated at $95^{\circ} \mathrm{C}$ for $3 \mathrm{~min}$ before thermal cycling at $95^{\circ} \mathrm{C}$ for $10 \mathrm{sec}$ and $56^{\circ} \mathrm{C}$ for $45 \mathrm{sec}$. Reactions were performed in triplicate. Relative gene expression was calculated by comparing the number of thermal cycles that were necessary to generate threshold amounts of product (CT) as described previously $^{26}$. CT was calculated for the genes of interest and for the housekeeping gene $\beta$-actin. For each cDNA sample, the CT for $\beta$-actin was subtracted from the CT for each gene of interest to give 
the parameter $\Delta \mathrm{CT}$, thus normalising the initial amount of RNA used. The amount of each target was was calculated as $2^{-\Delta \Delta \mathrm{CT}}$, where $\Delta \Delta \mathrm{CT}$ is the difference between the $\Delta \mathrm{CT}$ of the two cDNA samples to be compared.

Detection of MKP-1 protein in cultured cells MKP-1 expression in cultured cells was measured by immunostaining of methanol-fixed cells using rabbit anti-MKP-1 antibodies and Alexafluor 488conjugated secondary antibodies and Draq-5 followed by laser-scanning confocal microscopy (LSM 510 META; Zeiss, Oberkochen, Germany). Image analysis was performed using Velocity software (Improvision, Coventry, UK) to calculate average fluorescence values after subtracting background fluorescence values from cells stained with secondary antibody alone. Alternatively, levels of MKP-1 were measured in cytosolic or nuclear lysates prepared using the NucBuster kit (Novagen, San Diego, CA, USA) by Western blotting using anti-MKP-1 antibodies, HRP-conjugated secondary antibodies and chemiluminescent detection.

Statistics Differences between samples were analysed using a paired Student's t-test $\left({ }^{*} \mathrm{p}<0.05\right.$, $\left.{ }^{* *} \mathrm{p}<0.01, * * * \mathrm{p}<0.001\right)$ 


\section{RESULTS}

Endothelial VCAM-1 expression is suppressed in a protected region of the aorta We observed by en face immunostaining that basal expression of VCAM-1 in untreated animals was significantly lower in EC at the greater curvature of the aortic arch (LP region) compared to the lesser curvature (HP region) (Fig. 1, compare 1 and 2). Injection of LPS induced high levels of VCAM-1 in the HP region of the mouse aorta (compare 2 and 4) whereas EC in the LP region were relatively refractory to pro-inflammatory activation (compare 3 and 4). Thus our data confirm previous reports that both constitutive and LPS-inducible activation of EC is suppressed in EC in the LP region which is protected from atherosclerosis ${ }^{17,18}$.

\section{Activation of p38 and JNK MAP kinases in EC is suppressed in a protected region of the aorta}

To assess the activity of pro-inflammatory MAP kinases in EC at HP and LP sites we performed en face immunostaining using antibodies that recognise phosphorylated forms of p38 or JNK. In untreated animals, phosphorylated p38 was detected in the HP region but was not identified in the LP region (Fig. 2, compare 1 and 2). LPS treatment elevated phosphorylated p38 levels by approximately two-fold in the HP region (compare 2 and 4) but did not generate detectable levels of phospho-p38 in the LP site (compare 3 and 4). The suppression of phosphorylated p38 in the LP region was not caused by reduced protein expression of p38 which was similar in HP and LP sites (Supplementary Fig. 2). In parallel studies, we observed that phosphorylated JNK was expressed in HP regions of untreated animals and was induced strongly by LPS treatment (Fig. 3, compare 2 and 4). In contrast, phosphorylated JNK was not detected in LP regions of either untreated or LPS-treated animals (compare 1 and $3 \& 2$ and 4). These observations could not be attributed to differences in JNK expression which was similar in HP and LP regions (data not shown). Thus we conclude that activation of p38 and JNK by phosphorylation in EC occurs constitutively and can be enhanced by LPS treatment at HP sites but is suppressed in LP regions. 
Expression of MKP-1 in EC exposed to high shear stress Computational fluid dynamic models suggest that the greater curvature of the murine aorta (containing the LP region) is exposed to higher shear stresses than the lesser curvature (containing the HP region) ${ }^{21}$. Thus we hypothesized that high shear may suppress p38 and JNK in LP regions by inducing negative regulators of MAP kinases, e.g. MKPs. This was tested using cultured HUVEC which were exposed either to high shear stress (12 dynes $/ \mathrm{cm}^{2}$ for $24 \mathrm{~h}$ ) or to static conditions as a control. We validated the approach by demonstrating that shear stress can induce hemeoxygenase-1 mRNA and proteins (Figs. 4A and 4D) as reported previously $^{28}$. Shear stress also induced MKP-1 transcripts in cultured HUVEC but had little or no effect on MKP-2 or MKP-3 (Fig. 4B). The effects of shear stress on MKP-1 transcripts were paralleled by increases in MKP-1 protein levels in HUVEC as demonstrated by immunostaining (Fig. 4C) or by western blotting (Fig. 4D) using anti-MKP-1 antibodies.

We used en face staining using anti-MKP-1 antibodies to examine whether MKP-1 expression in aortic EC correlated with local hemodynamics in vivo. Analysis of wild-type C57BL/6 mice revealed that MKP-1 was expressed at high levels in EC in the LP region but only at low levels in the HP region (Fig. 5). Staining was not observed in parallel experiments using MKP-1/- $(\mathrm{C} 57 \mathrm{BL} / 6)$ animals (data not shown), indicating that the anti-MKP-1 antibodies were specific for MKP-1. Thus we conclude that MKP-1 is expressed at elevated levels in EC exposed to high shear in vivo and suggest that it may suppress MAPK activities and pro-inflammatory activation in protected regions of the aorta.

MKP-1 is necessary for suppression of endothelial activation in a protected region of the aorta We used MKP-1 ${ }^{-/}$mice to examine whether MKP-1 is necessary for suppression of EC activation in LP regions. In contrast to wild-type animals, EC in LP regions of $\mathrm{MKP}-\mathrm{1}^{-/-}$animals expressed constitutively phosphorylated forms of p38 and JNK that could be induced further by LPS treatment (compare Fig. 6, panels 1 and 3 with Fig. 2; compare Fig. 7, panels 1 and 3 with Fig. 3). The 
restoration of p38 and JNK phosphorylation by genetic deletion of MKP-1 could not be attributed to differences in expression of either p38 or JNK which were detected at similar levels in $\mathrm{MKP}^{-/-}$and wild-type mice (Supplementary Fig. 2 and data not shown). Thus we conclude that MKP-1 is essential for the inhibition of p38 and JNK activation at the LP site. We observed that active, phosphorylated forms of p38 or JNK were expressed at similar levels in EC at HP sites of wild-type and MKP-1 ${ }^{-/}$ animals (compare Fig. 6, panels 2 and 4 with Fig. 2; compare Fig. 7, panels 2 and 4 with Fig. 3), a finding that is consistent with our observation that MKP-1 is expressed at low levels or is absent from EC in the HP region of wild-type mice (Fig. 5). Interestingly levels of phosphorylated p38 and JNK were approximately 2-fold higher in the HP region compared to the LP region of MKP-1 ${ }^{-/-}$mice (Fig. 6, compare 3 and 4; Fig. 7, compare 3 and 4) suggesting that other negative regulators co-operate with MKP-1 to suppress MAP kinase activities at the LP site.

In contrast to wild-type animals, en face staining revealed that VCAM-1 was induced by LPS at similar levels in LP and HP regions of MKP1 $1^{-/-}$animals (compare Fig. 8, panels 3 and 4 with Fig. 1). Thus we conclude that MKP-1 is necessary for suppression of VCAM-1 expression at the LP site.

To test directly whether MKP-1 expression in the vessel wall is required for suppression of EC activation in LP regions we isolated aortae from wild-type and MKP-1 ${ }^{-/-}$animals and examined their responses to LPS treatment ex vivo. We observed by en face staining that the application of LPS significantly elevated levels of phosphorylated p38 in the LP site in aortae from MKP1 $1^{-/}$animals whereas the LP site in aortae from wild-type mice was refractory to stimulation (Fig. 9, compare 3 and 7). Furthermore, VCAM-1 was induced by LPS at similar levels in LP and HP sites in aortae of MKP-1 ${ }^{-/-}$mice but was suppressed in LP sites in aortae of wild-type animals (Fig. 10, compare 3 and 7). These observations therefore indicate that vascular MKP-1 is required for suppression of EC activation at the LP region. 


\section{DISCUSSION}

The adhesion protein VCAM-1 can be induced in EC by pro-inflammatory stimuli and plays a key role in the atherogenic process by recruiting monocytes and T-lymphocytes to vessel walls ${ }^{4,5}$. Here we report that endothelial expression of VCAM-1 in response to LPS is suppressed in EC exposed to high shear stress in the greater curvature of the aorta, a finding that is consistent with previous reports $^{17,18}$. Thus reduced expression of VCAM-1 provides a potential explanation for the protection of this region of the arterial tree from vascular inflammation and atherosclerosis.

Pro-inflammatory mediators activate p38 and JNK which play an essential role in VCAM-1 expression by activating ATF-2 and c-jun transcription factors and by regulating VCAM-1 mRNA stability ${ }^{11,12,14}$. Here we demonstrate that activation of p38 and JNK in EC occurs constitutively and can be enhanced by LPS treatment in the lesser curvature of the aortic arch, a region that is exposed to low shear and is pre-disposed to vascular inflammation and atherosclerosis. Given the role of p38 and JNK in regulating the expression of pro-inflammatory proteins it is likely that their activation plays a key part in regulating vascular inflammation at regions of the aorta that are susceptible to atherosclerosis. This concept is supported by previous studies demonstrating that a pharmacological inhibitor of JNK can reduce lesion formation in an atherosusceptible mouse strain ${ }^{29}$.

We observed that activation of p38 and JNK in EC was suppressed at the greater curvature, a protected site that is exposed to high shear. Our findings are supported by previous studies which revealed that high shear stress can suppress the activation of JNK, p38 and downstream transcription factors and inhibit the induction of VCAM-1 and other adhesion proteins in cultured EC or in arteries perfused ex vivo 22, 25-27, 30, 31 Thus it is likely that suppression of p38 and JNK activities in EC exposed to high shear stress plays a vital role in inhibiting inflammation and atherosclerosis in protected regions of the arterial tree. In addition to cellular activation, p38 and JNK are known to regulate several other fundamental physiological activities in EC including proliferation, apoptosis 
and migration ${ }^{32,33}$. It will therefore be of interest in future studies to assess the influence of the spatial distribution of p38 and JNK activities in the arterial tree on the regulation of these processes.

We sought the molecular mechanism for suppression of p38 and JNK activation in a protected region of the aorta by examining the effects of prolonged shear stress on the expression of negative regulators of MAPKs in cultured EC. We observed that the application of high shear to EC led to the induction of MKP-1, an intracellular protein that is known to repress endothelial activation by inhibiting the activities of both p38 and $\mathrm{JNK}^{6}$. This finding was validated in vivo by studies of the murine aorta which demonstrated that MKP-1 was expressed in EC in regions exposed to high shear but was absent at adjacent sites exposed to relatively low shear. Several signaling pathways have been shown to upregulate MKP-1 in EC ${ }^{34-37}$ and further work is now required to identify whether they participate in the induction of MKP-1 in response to shear stress. The VEGFR pathway is an interesting candidate because it is known to regulate endothelial responses to shear ${ }^{19}$ and can also induce MKP-1 transcription ${ }^{37}$.

We observed that activation of p38 and JNK and expression of VCAM-1 were restored in EC at an atheroprotected (high shear) region of the aorta by genetic deletion of MKP-1, and therefore conclude that MKP-1 is required for suppression of EC activation at this site. To assess the role of MKP-1 expression in the vasculature directly we isolated aortae from wild-type or MKP-1 ${ }^{-/-}$mice and compared their responses to LPS ex vivo. Studies of aortae from wild-type animals revealed that p38 and JNK activation and expression of VCAM-1 was suppressed in an atheroprotected region treated with LPS ex vivo. These data suggest that the protective effects of high shear stress persist for several hours when vessels are exposed to static conditions, a concept that is consistent with previous studies of the responses of cultured EC to shear ${ }^{27}$. In contrast, parallel studies of aortae from MKP-1 ${ }^{-/-}$mice demonstrated that EC in an atheroprotected region were sensitive to LPS treatment which activated p38 and JNK and induced high levels of VCAM-1. Thus we conclude that MKP-1 expression in the 
vessel wall is required for the suppression of EC activation at atheroprotected sites.

Our findings suggest that the mechanism by which high shear protects arteries from inflammation relies on the establishment of persistent endothelial expression of MKP-1 which suppresses cellular activation by inhibiting the activities of p38 and JNK. Further work is required to examine whether MKP-1 protects arteries from atherogenesis in response to hypercholesterolemia. Our findings complement previous studies demonstrating that EC at atheroprotected regions express reduced levels of pro-inflammatory Toll-like receptors and NF- $\kappa \mathrm{B}$ transcription factors ${ }^{17,38}$. Thus the novel findings presented herein and those from previous studies ${ }^{17,38}$ suggest that regions of the arterial tree exposed to high shear are protected from pro-inflammatory activation by suppression of both MAPK and NFкB signalling pathways.

\section{ACKNOWLEDGEMENTS}

The study was funded by the British Heart Foundation. 


\section{FIGURE LEGENDS}

Fig. 1 VCAM-1 expression in EC is suppressed in a protected region of the aorta VCAM-1 expression levels were assessed in HP and LP regions of the aorta of C57BL/6 mice which were treated with LPS $(8 \mathrm{mg} / \mathrm{kg}$ for $6 \mathrm{~h} ; \mathrm{n}=4)$ or remained untreated as controls $(n=4)$. VCAM-1 was detected by en face staining using anti-VCAM-1 antibodies and AlexaFluor568-conjugated secondary antibodies (red) followed by confocal microscopy. EC were identified by co-staining with anti-CD31 antibodies conjugated to FITC (green). Cell nuclei were identified using Draq5 (purple). Representative images are shown (upper panels). VCAM-1 expression was quantified by measuring fluorescence from multiple cells (>100) in HP and LP regions of treated or untreated mice. Mean fluorescence intensities are shown with standard deviations (lower panel). Differences between samples were analysed using an unpaired t-test.

Fig. 2 Phosphorylation of p38 in EC is suppressed in a protected region of the aorta The levels of phosphorylated p38 were assessed in HP and LP regions of the aorta of C57BL/6 mice which were treated with LPS (8mg/kg for $1 \mathrm{~h} ; \mathrm{n}=4)$ or remained untreated as controls $(\mathrm{n}=4)$. Phosphorylated p38 was detected by en face staining using anti-phos-p38 antibodies and AlexaFluor568-conjugated secondary antibodies (red) followed by confocal microscopy. EC were identified by co-staining with anti-CD31 antibodies conjugated to FITC (green). Cell nuclei were identified using Draq5 (purple). Representative images are shown (upper panels). Levels of phosphorylated p38 were quantified by measuring fluorescence from multiple cells (>100) in HP and LP regions of treated or untreated mice. Mean fluorescence intensities are shown with standard deviations (lower panel). Differences between samples were analysed using an unpaired t-test.

Fig. 3 Phosphorylation of JNK in EC is suppressed in a protected region of the aorta The levels of phosphorylated JNK were assessed in HP and LP regions of the aorta of C57BL/6 mice which were 
treated with LPS (8mg/kg for $1 \mathrm{~h} ; \mathrm{n}=4)$ or remained untreated as controls $(\mathrm{n}=4)$. Phosphorylated JNK was detected by en face staining using anti-phos-JNK antibodies and AlexaFluor568-conjugated secondary antibodies (red) followed by confocal microscopy. EC were identified by co-staining with anti-CD31 antibodies conjugated to FITC (green). Cell nuclei were identified using Draq5 (purple). Representative images are shown (upper panels). Levels of phosphorylated JNK were quantified by measuring fluorescence from multiple cells (>100) in HP and LP regions of treated or untreated mice. Mean fluorescence intensities are shown with standard deviations (lower panel). Differences between samples were analysed using an unpaired t-test.

Fig. 4 Shear stress induces MKP-1 in cultured EC HUVEC were exposed to shear stress (12 dynes $/ \mathrm{cm}^{2}$ ) for $24 \mathrm{~h}$ or were cultured under static conditions. (A, B) Levels of particular transcripts were quantified by real-time PCR using gene-specific PCR primers. The amount of each target transcript was normalised by measuring $\beta$-actin transcript levels. Mean values calculated from triplicate measurements are shown with standard deviations. Differences between samples were analysed using a paired t-test. (C) Fixed cells were assessed by immunostaining using anti-MKP-1 antibodies and fluorescent secondary antibodies followed by confocal microscopy. Cell nuclei were identified using Draq5 (purple). Representative images are shown (left panels). MKP-1 protein levels were quantified using image analysis software and are presented as mean values pooled from two experiments with standard deviations (right panels). (D) Expression levels of HO-1 or MKP-1 proteins were determined by Western blotting of cell lysates using specific antibodies and were normalised by Western blotting using anti-tubulin antibodies.

Fig. $5 \mathrm{MKP}-1$ is expressed in EC in a protected region of the aorta MKP-1 expression levels were assessed in HP and LP regions of the aorta of C57BL/6 mice by en face staining using anti-MKP-1 antibodies and AlexaFluor568-conjugated secondary antibodies (red) followed by confocal microscopy. EC were identified by co-staining with anti-CD31 antibodies conjugated to FITC (green). 
Cell nuclei were identified using Draq5 (purple). Representative images are shown (upper panels). Levels of MKP-1 were quantified by measuring fluorescence from multiple cells (>100) in HP and LP regions. Mean fluorescence intensities are shown with standard deviations (lower panel). Differences between samples were analysed using an unpaired t-test.

Fig. 6 MKP-1 suppresses p38 activation in EC in a protected region of the aorta The levels of phosphorylated p38 were assessed in HP and LP regions of the aorta of MKP-1 ${ }^{-/}$mice which were treated with LPS (8mg/kg for $1 \mathrm{~h} ; \mathrm{n}=4)$ or remained untreated as controls $(\mathrm{n}=4)$. Phosphorylated p38 was detected by en face staining using anti-phos-p38 antibodies and AlexaFluor568-conjugated secondary antibodies (red) followed by confocal microscopy. EC were identified by co-staining with anti-CD31 antibodies conjugated to FITC (green). Cell nuclei were identified using Draq5 (purple). Representative images are shown (upper panels). Levels of phosphorylated p38 were quantified by measuring fluorescence from multiple cells $(>100)$ in HP and LP regions of treated or untreated mice. Mean fluorescence intensities are shown with standard deviations (lower panel). Differences between samples were analysed using an unpaired t-test.

Fig. 7 MKP-1 suppresses JNK activation in EC in a protected region of the aorta The levels of phosphorylated JNK were assessed in HP and LP regions of the aorta of MKP-1/- mice which were treated with LPS (8mg/kg for $1 \mathrm{~h} ; \mathrm{n}=4)$ or remained untreated as controls $(\mathrm{n}=4)$. Phosphorylated JNK was detected by en face staining using anti-phos-JNK antibodies and AlexaFluor568-conjugated secondary antibodies (red) followed by confocal microscopy. EC were identified by co-staining with anti-CD31 antibodies conjugated to FITC (green). Cell nuclei were identified using Draq5 (purple). Representative images are shown (upper panels). Levels of phosphorylated JNK were quantified by measuring fluorescence from multiple cells $(>100)$ in HP and LP regions of treated or untreated mice. Mean fluorescence intensities are shown with standard deviations (lower panel). Differences between samples were analysed using an unpaired t-test. 
Fig. 8 MKP-1 suppresses VCAM-1 expression in EC in a protected region of the aorta VCAM-1 expression was assessed in the LP region of the aorta of MKP-1 ${ }^{-/-}$mice which were treated with LPS $(8 \mathrm{mg} / \mathrm{kg}$ for $6 \mathrm{~h} ; \mathrm{n}=4)$ or remained untreated as controls $(\mathrm{n}=4)$ by en face staining using anti-VCAM-1 antibodies and AlexaFluor568-conjugated secondary antibodies (red) followed by confocal microscopy. EC were identified by co-staining with anti-CD31 antibodies conjugated to FITC (green). Cell nuclei were identified using Draq5 (purple). Representative images are shown (upper panels). VCAM-1 levels were quantified by measuring fluorescence from multiple cells $(>100)$ in treated or untreated mice. Mean fluorescence intensities are shown with standard deviations (lower panel). Differences between samples were analysed using an unpaired t-test.

Fig. 9 Vascular MKP-1 expression suppresses p38 activation in EC in a protected region of the aorta The levels of phosphorylated p38 were assessed in HP and LP regions of the aorta of wild-type or $\mathrm{MKP}-1^{-/-}$mice. Aortae were harvested and then bathed in cell culture medium for $1 \mathrm{~h}$ either in the presence or absence of LPS (10 $\mu \mathrm{g} / \mathrm{ml}$ for $1 \mathrm{~h} ; \mathrm{n}=4$ per group). Phosphorylated p38 was detected by en face staining using anti-phos-p38 antibodies and AlexaFluor568-conjugated secondary antibodies (red) followed by confocal microscopy. EC were identified by co-staining with anti-CD31 antibodies conjugated to FITC (green). Cell nuclei were identified using Draq5 (purple). Representative images are shown (upper panels). Levels of phosphorylated p38 were quantified by measuring fluorescence from multiple cells (>100) in HP and LP regions of treated or untreated vessels. Mean fluorescence intensities are shown with standard deviations (lower panel). Differences between samples were analysed using an unpaired t-test.

Fig. 10 Vascular MKP-1 expression suppresses VCAM-1 expression in EC in a protected region of the aorta VCAM-1 expression levels were assessed in HP and LP regions of the aorta of wildtype or MKP-1/- mice. Aortae were harvested and then bathed in cell culture medium for $6 \mathrm{~h}$ either in 
the presence or absence of LPS (10 $\mu \mathrm{g} / \mathrm{ml}$ for $1 \mathrm{~h}$; $\mathrm{n}=4$ per group). VCAM-1 was detected by en face staining using anti-VCAM-1 antibodies and AlexaFluor568-conjugated secondary antibodies (red) followed by confocal microscopy. EC were identified by co-staining with anti-CD31 antibodies conjugated to FITC (green). Cell nuclei were identified using Draq5 (purple). Representative images are shown (upper panels). Levels of VCAM-1 were quantified by measuring fluorescence from multiple cells $(>100)$ in HP and LP regions of treated or untreated vessels. Mean fluorescence intensities are shown with standard deviations (lower panel). Differences between samples were analysed using an unpaired t-test. 
Reference List

1. Libby,P. Inflammation in atherosclerosis. Nature 420, 868-874 (2002).

2. Huo,Y. \& Ley,K. Adhesion molecules and atherogenesis. Acta Physiologica Scandinavica 173, 35-43 (2001).

3. Kol,A. \& Libby,P. The mechanisms by which infectious agents may contribute to atherosclerosis and its clinical manifestations. Trends in Cardiovascular Medicine 8, 191-199 (1998).

4. Dansky,H.M., Barlow,C.B., Cybulsky,M.I., \& Smith,J.D. Decreased endothelial VCAM-1 expression reduces monocyte adherence and atherosclerosis in apolipoprotein E-deficient mice. Circulation 102, 114 (2000).

5. Cybulsky,M.I. et al. A major role for VCAM-1, but not ICAM-1, in early atherosclerosis. Journal of Clinical Investigation 107, 1255-1262 (2001).

6. Wadgaonkar,R. et al. Regulation of c-Jun N-terminal kinase and p38 kinase pathways in endothelial cells. American Journal of Respiratory Cell and Molecular Biology 31, 423-431 (2004).

7. Schieven,G.L. The biology of p38 kinase: A central role in inflammation. Current Topics in Medicinal Chemistry 5, 921-928 (2005).

8. $\quad$ Kuida,K. \& Boucher,D.M. Functions of MAP kinases: Insights from gene-targeting studies. Journal of Biochemistry 135, 653-656 (2004).

9. Dong,C., Davis,R.J., \& Flavell,R.A. MAP kinases in the immune response. Annual Review of Immunology 20, 55-72 (2002).

10. Hoefen,R.J. \& Berk,B.C. The role of MAP kinases in endothelial activation. Vascular Pharmacology 38, 271-273 (2002).

11. Bayat,H., Xu,S.Q., Pimentel,D., Cohen,R.A., \& Jiang,B.B. Activation of thromboxane receptor upregulates interleukin (IL)-1 beta-induced VCAM-1 expression through JNK signaling. Arteriosclerosis Thrombosis and Vascular Biology 28, 127-134 (2008).

12. Ahmad,M., Theofanidis,P., \& Medford,R.M. Role of activating protein-1 in the regulation of the vascular cell adhesion molecule-1 gene expression by tumor necrosis factor-alpha. Journal of Biological Chemistry 273, 4616-4621 (1998).

13. Collins, T. et al. Transcriptional Regulation of Endothelial-Cell Adhesion Molecules - NfKappa-B and Cytokine-Inducible Enhancers. Faseb Journal 9, 899-909 (1995).

14. Pietersma,A. et al. P38 mitogen activated protein kinase regulates endothelial VCAM-1 expression at the post-transcriptional level. Biochemical and Biophysical Research Communications 230, 44-48 (1997).

15. Liu,Y.S., Shepherd,E.G., \& Nelin,L.D. MAPK phosphatases - regulating the immune 
response. Nature Reviews Immunology 7, 202-212 (2007).

16. Cunningham,K.S. \& Gotlieb,A.I. The role of shear stress in the pathogenesis of atherosclerosis. Laboratory Investigation 85, 9-23 (2005).

17. Hajra,L. et al. The NF-kappa B signal transduction pathway in aortic endothelial cells is primed for activation in regions predisposed to atherosclerotic lesion formation. Proceedings of the National Academy of Sciences of the United States of America 97, 9052-9057 (2000).

18. Iiyama,K. et al. Patterns of vascular cell adhesion molecule-1 and intercellular adhesion molecule-1 expression in rabbit and mouse atherosclerotic lesions and at sites predisposed to lesion formation. Circulation Research 85, 199-207 (1999).

19. Tzima,E. et al. A mechanosensory complex that mediates the endothelial cell response to fluid shear stress. Nature 437, 426-431 (2005).

20. Dimmeler,S. et al. Activation of nitric oxide synthase in endothelial cells by Aktdependent phosphorylation. Nature 399, 601-605 (1999).

21. Suo,J. et al. Hemodynamic shear stresses in mouse aortas - Implications for atherogenesis. Arteriosclerosis Thrombosis and Vascular Biology 27, 346-351 (2007).

22. Dai,G.H. et al. Distinct endothelial phenotypes evoked by arterial waveforms derived from atherosclerosis-susceptible and -resistant regions of human vasculature. Proceedings of the National Academy of Sciences of the United States of America 101, 14871-14876 (2004).

23. Cheng,C. et al. Shear stress affects the intracellular distribution of eNOS: direct demonstration by a novel in vivo technique. Blood 106, 3691-3698 (2005).

24. Lin,K. et al. Molecular mechanism of endothelial growth arrest by laminar shear stress. Proceedings of the National Academy of Sciences of the United States of America 97, 9385-9389 (2000).

25. Yamawaki,H., Lehoux,S., \& Berk,B.C. Chronic physiological shear stress inhibits tumor necrosis factor-induced proinflammatory responses in rabbit aorta perfused ex vivo. Circulation 108, 1619-1625 (2003).

26. Partridge,J. et al. Laminar shear stress acts as a switch to regulate divergent functions of NF-kappa B in endothelial cells. Faseb Journal 21, 3553-3561 (2007).

27. Chiu,J.J. et al. Shear stress increases ICAM-1 and decreases VCAM-1 and E-selectin expressions induced by tumor necrosis factor-alpha in endothelial cells. Arteriosclerosis Thrombosis and Vascular Biology 24, 73-79 (2004).

28. De Keulenaer,G.W. et al. Oscillatory and steady laminar shear stress differentially affect human endothelial redox state - Role of a superoxide-producing NADH oxidase. Circulation Research 82, 1094-1101 (1998).

29. Ricci,R. et al. Requirement of JNK2 for scavenger receptor A-mediated foam cell formation in atherogenesis. Science 306, 1558-1561 (2004).

30. Yamawaki,H., Pan,S., Lee,R.T., \& Berk,B.C. Fluid shear stress inhibits vascular inflammation by decreasing thioredoxin-interacting protein in endothelial cells. Journal of Clinical Investigation 115, $733-738$ (2005). 
31. Hojo,Y. et al. Fluid shear stress attenuates hydrogen peroxide-induced c-Jun NH2terminal kinase activation by a thioredoxin reductase-mediated mechanism. Circulation 104, 65 (2001).

32. Liu,J., Minemoto,Y., \& Lin,A.N. c-Jun N-terminal protein kinase 1 (JNK1), but not JNK2, is essential for tumor necrosis factor alpha-induced c-Jun kinase activation and apoptosis. Molecular and Cellular Biology 24, 10844-10856 (2004).

33. McMullen,M.E., Bryant,P.W., Glembotski,C.C., Vincent,P.A., \& Pumiglia,K.M. Activation of p38 has opposing effects on the proliferation and migration of endothelial cells. Journal of Biological Chemistry 280, 20995-21003 (2005).

34. Li,M.X., Zhou,J.Y., Ge,Y.B., Matherly,L.H., \& Wu,G.S. The phosphatase MKP1 is a transcriptional target of p53 involved in cell cycle regulation. Journal of Biological Chemistry 278, 41059-41068 (2003).

35. Chi,H.B. et al. Dynamic regulation of pro- and anti-inflammatory cytokines by MAPK phosphatase 1 (MKP-1) in innate immune responses. Proceedings of the National Academy of Sciences of the United States of America 103, 2274-2279 (2006).

36. Lasa,M., Abraham,S.M., Boucheron,C., Saklatvala,J., \& Clark,A.R. Dexamethasone causes sustained expression of mitogen-activated protein kinase (MAPK) phosphatase 1 and phosphatase-mediated inhibition of MAPK p38. Molecular and Cellular Biology 22, 7802-7811 (2002).

37. Kinney,C.M., Chandrasekharan,U.M., Mavrakis,L., \& DiCorleto,P.E. VEGF and thrombin induce MKP-1 through distinct signaling pathways: role for MKP-1 in endothelial cell migration. American Journal of Physiology-Cell Physiology 294, C241-C250 (2008).

38. Mullick,A.E. et al. Increased endothelial expression of Toll-like receptor 2 at sites of disturbed blood flow exacerbates early atherogenic events. Journal of Experimental Medicine 205, 373-383 (2008). 